\title{
Comparison Analysis of EPMS and TABU/PSO EPMS Routing Protocol in WSN \\ Sannia
}

\author{
Research Scholar, Department of Computer Engineering \\ CT Institute of Engineering and Management Technology \\ Jalandhar, Punjab, India \\ swtsanniajoshi@gmail.com \\ Varsha \\ Assistant professor, Department of Computer Engineering \\ CT Institute of Engineering and Management Technology \\ Jalandhar, Punjab, India \\ barkhabright@gmail.com
}

\begin{abstract}
Routing in wireless sensor network is a key challenge. The appropriate routing is done in wsn by using swarm intelligence approach. Various swarm intelligence techniques are available like ant colony optimization, particle swarm optimization, artificial bee colony optimization etc. But hybrid approach of TABU and PSO is a promising one. The integration of TS to PSO allows algorithm to sustain the population diversity and avoiding directing to misguiding local optima. Average energy consumption is high in TS as comparison with PSO and less calculation time is utilized in TS than PSO. The output of the experiment show that the optimize algorithm not only reduce the number of paths but also finding the shortest path at the place of largest path. The simulation result shows that combination of TABU-PSO performs better than Energy Efficient PSO in terms of dead nodes, alive nodes and throughput of the network.
\end{abstract}

Keywords: Wireless Sensor Networks, routing, TABU/PSO dead nodes, alive nodes and throughput.

\section{INTRODUCTION}

A wireless device network with an outsized quantity of tiny device nodes may be utilized as an efficient tool for gathering knowledge in numerous circumstances. One among the most problems in wireless device networks is producing an energy-efficient routing protocol that has a substantial effect on the overall lifespan of the sensor network. Sensor nodes evaluate the ambient circumstances from the natural world surrounding them. The applications of WSN are numerous from health monitoring to battle field. The pattern of remote sensing has become greatly modified by useful and low-priced sensors as well as demanded software bundles. To boot, client can supervise and ensure the fundamental natural world from remote location. Lots of routing, power management, and data dissemination protocols have been generally planned for WSNs where energy consciousness is important design issue. Routing protocols in WSNs might dissent depending on the application and network architecture. Wireless sensor network (WSN) area unit extremely distributed networks of little, light-weight wireless nodes, deployed in massive numbers to watch the setting or system by the measure of parameters resembling temperature, pressure wetness, sound, vibration, pollutants and put together relay their detected information to the sink node. Each node in the network associated with one another. Each sensor node within the network consists of three subsystems: 1) The device system that is employed to sense the environment,

2)The process system that performs

the native computations on the perceived knowledge.[1,2]

3) The communication system that is answerable

for sharing the perceived knowledge with the neighbouring device nodes.

Amongst the sources of energy usage in a sensor node, wireless data transmission is the most vital. Within a clustering organization, intra-cluster communication can be single hop or multihop, as well as inter-cluster communication. Multihop communication within a data source and a data sink is usually more energy effective than direct transmission because of the features of wireless channel [3]. However, the hot-spots problem arises when using the multihop forwarding model in inter-cluster communication. Because the cluster heads nearer to the data sink are loaded down with large relay traffic, they will expire much quicker than the other cluster heads, cutting down detection coverage and getting network segmentation .

The rest of the paper is prepared as follows. Section II presents an overview of energy efficient PSO. Section III presents TABU search. Section IV gives the simulation results and analysis and Section V concludes the work.

\section{ENERGY EFFICIENT PSO (EPMS)}

The main aim of this protocol is to conserve the energy in communication to prolong the network lifespan. Energy efficient PSO based routing algorithm with Mobile Sink 
support for WSNs, which we name it EPMS for short. EPMS routing algorithm mainly combines the virtual clustering and mobile sink techniques during routing process. [4]

Firstly, it uses the PSO algorithm to divide the network into several regions. In each region, the EPMS uses a similar clustering algorithm to select the cluster head nodes inside each cluster. It combines with the two conditions of the region of the gravity center of the distance and the energy of the node.

Then, the EPMS defines three kinds of data packet formats: Hello, Message-s and Message-h packets. The Hello packet is used to determine which cluster area send data to the mobile sink. The Message-s packet sends data to the sink node, and the Message-h sends information to the cluster head. EPMS can balance energy consumption, prolongs network lifetime and reduces the transmission delay based on the extensive simulation results [5,6,7]. Several algorithms for optimization has to be considered like TABU SEARCH and PSO, ACO and Genetic algorithm.

\section{TABU SEARCH}

Tabu Search Optimization is a system which gives the arrangement when the ideal esteem is stuck into nearby optima .In this convention, we have considered TSO for the group head choice so that $\mathrm{CH}$ can be chosen proficiently. All the more critically as TSO doesn't return to its past arrangement, comparably once the hub is chosen as $\mathrm{CH}$, there are clear quantities of rounds and pivot of $\mathrm{CH}$ happen before a similar hub is doled out the job of CH.TSO refreshes the Tabu rundown by evacuating the as of now acquired arrangements along these lines it center around different arrangements. Likewise the $\mathrm{CH}$ hubs which are chosen as the $\mathrm{CH}$ they are expelled from the rundown of the hubs which are yet to progress toward becoming bunch heads. TS are regularly pondered as a speculation of monotonous upgrades like state army. It's viewed as an adaptative methodology having the adaptability to utilize a few different ways, as connected science calculations and specific heuristics that it manual for beat the limitations of local optimality. TS depends on thoughts that might be utilized in every software engineering and improvement fields. Throughout the days TS was better by a few analysts to suit one among the diverse mainstream key methodology. Cutting plane methodology, remain in requirement and steepest climb are monstrous achievement inside the improvement of TS. Tabu Search apply limits to guide the inquiry to various areas. These limitations are in regard of the $\mathrm{m} / \mathrm{m}$ structures that might be thought of as clever capabilities. Insight needs adaptative memory and responsive investigation .Eg:, while climb a stack one recollect (versatile memory) property of strategies s/he has voyage and settles on vital options (responsive investigation) on the gratitude to achieve your pinnacle or plunge. TS conjointly utilizes agreeable examination because of an awful key call could offer a great deal of information than a genuine arbitrary one to return up with quality arrangements. TS have memory property that recognizes it from various hunt styles. It's adaptative memory that is conjointly totally unique in relation to inflexible memory utilized by branch and beyond any doubt techniques. Memory in TS has four measurements: quality, recency, recurrence, and impact. TS power a move to a neighbor with least esteem crumbling. TS utilizes memory to remain track of answers aforesaid visited so it will hinder returning to that arrangement. Memory-based systems are sign of TS approaches a few applications don't grasp propelled highlights of TS since reasonable arrangements are for the most part accomplished by simple styles. A fundamental tabu look calculation for an expansion disadvantage $[8,9]$.

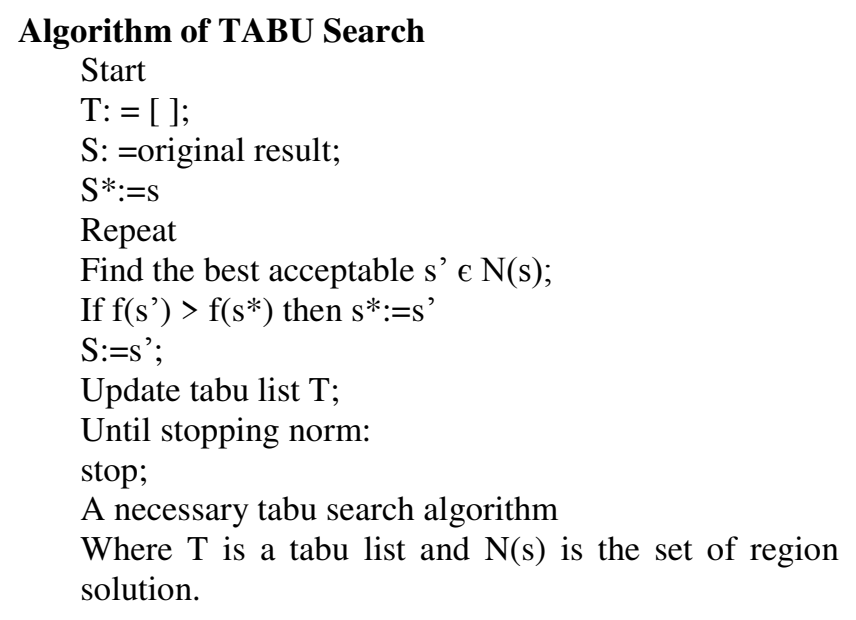

\section{Advantages of Tabu Search}

1. It is intuitive, since it typically doesn't embody random parts.

2. it's settled, and chooses the most effective choice obtainable to boost a solution.

\section{Particle Swarm Optimization (PSO)}

In 1995, Dr. Eberhart and Dr. Kennedy proposed Particle Swarm Optimization (PSO) system. This strategy emulates the social conduct of feathered creature rushing. Give us a chance to consider a case of feathered creatures that are set haphazardly in a territory and that zone has just a bit of sustenance being looked [10]. The area of sustenance isn't known by the flying creatures. They just know the separation of nourishment from them. For this situation, the least complex system to get sustenance is to pursue that winged creature which is nearer to nourishment. Fowl is classified "molecule". The particles are having a few qualities which can be determined utilizing wellness work and certain speed. In it each molecule refreshes "best" two qualities i.e. best arrangement (wellness) called pbest and gbest. Utilizing these two qualities, particles refresh their speeds and positions as appeared in conditions.

$\operatorname{vel}[]=\operatorname{vel}[]+\mathrm{c} 1 * \operatorname{ran}() *(\operatorname{pbest}[]-\operatorname{present}[])+\mathrm{c} 2 * \operatorname{ran}()$ $*($ gbest[] -present[])

$$
\operatorname{pre}[]=\operatorname{per}[]+\operatorname{vel}[]
$$


vel[] represents velocity of particle, per[] represents current particle, ran () represents random number ranges from 0 to 1 and $\mathrm{c} 1, \mathrm{c} 2$ represents learning factors which is equal to $\mathrm{c} 1=\mathrm{c} 2$.

\section{Hybrid TABU/PSO optimization}

An algorithm based on TS and PSO is proposed for optimizing the routing in Wireless Sensor Network. PSO hold benefits such as greater convergence, resolving optimization efficiently, minimized population diversity and directing early converging towards a local optima. On other hand, TS is optimization method that could hypothetically congregate to global optimum solution, however it acquires more time span to attain near global minima The integration of TS to PSO allows algorithm to sustain the population diversity and avoiding directing to misguiding local optima. Average energy consumption is high in TS as comparison with PSO and less calculation time is utilized in TS than PSO. By combining both tradeoff between the two is avoided. Proposed TABUPSO shows the reduction of average packet rate and average end to end delay.

\section{Genetic Algorithm}

GA is adaptable heuristic interest estimation excited about organic procedure contemplations of regular assurance and innate characteristics. GA reproduces the survival of the fittest among people over persistent age for defying a trouble. Each age contain a universe of character strings that region unit intently looking like the body that we find in our deoxyribonucleic corrosive. Each individual addresses a degree in a very chase house and a possible game plan. The individual inside the world zone unit then mode to aptitude the methodology of improvement. GA depends on inherited structure and lead of chromosomes.

\begin{abstract}
Algorithm:
Above all else, the fundamental people is erratically made and new world zone unit made in resultant age misuse four critical frameworks that are referenced underneath.
\end{abstract}

Choice: picks individual for traverse and transformation.

Traverse: method that makes the trading of hereditary materials between people supply off springs.

Change: In corporate new hereditary trails GA keeps up this world and over and again modifies to make another period of chromosomes. This methodology is reiterated till most extraordinary amounts more seasoned territory unit achieved.

\section{Ant colony optimization (ACO)}

ACO is furthermore Member of swarm streamlining procedure, delivered by Marco Dorigo (1991 PhD proposition). It is fundamentally a technique to locate the most limited way among beginning spot and objective. It reflects the direct of trademark ants.

\section{Algorithm}

From the start ants moved haphazardly once sustenance source is found ants walk around to the state leave-taking markers (pheromones) that exhibit the way has found once totally various ants gone over these markers.

They are probably going to seek after a similar methodology with bound likelihood. On the off plausibility that they doing, they populate the methodology with their own markers as they pass on the sustenance back. The way gets more grounded a similar number of ants seek after a comparative way, as the ants drops pheromones each time they bring their sustenance. Then a couple of ants are still erratically chasing down closer nourishment source.

A similar strategy is used to find decide secure perfect solution for the voyaging sales rep issue. At the point when the sustenance asset is depleted the root has now not pheromones and consistently starts disintegrating.

\section{Simulation Results and Analysis}

\begin{tabular}{|l|l|}
\hline Area of simulation & $\begin{array}{l}100 \times 100 \\
\mathrm{~m}^{2}\end{array}$ \\
\hline Number of nodes & 100 \\
\hline $\begin{array}{l}C_{f s} \text { (energy used in short distant } \\
\text { communication) }\end{array}$ & $\begin{array}{l}10 \\
\mathrm{pJ} / \mathrm{bit} / \mathrm{m}^{2}\end{array}$ \\
\hline $\begin{array}{l}\epsilon_{m p} \text { (energy used in long distant } \\
\text { communication) }\end{array}$ & $\begin{array}{l}0.0013 \\
\mathrm{pJ} / \mathrm{bit} / \mathrm{m}^{4}\end{array}$ \\
\hline 1 (length of data) & $2000 \mathrm{bit}$ \\
\hline Ee (Initial energy of the nodes) & $0.5 \mathrm{~J}$ \\
\hline ET (Transmitting energy) & $50 \mathrm{~nJ} / \mathrm{bit}$ \\
\hline ER (Reception Energy) & $50 \mathrm{~nJ} / \mathrm{bit}$ \\
\hline $\begin{array}{l}\text { Ebf (Energy consumption in Beam } \\
\text { Forming) }\end{array}$ & $5 \mathrm{~nJ} / \mathrm{bit}$ \\
\hline
\end{tabular}

We have utilized MATLAB to tentatively check our results. The parameters utilized throughout the whole course of simulation have been recorded in Table 1 . Additionally, we have assessed EPMS and TP-EPMS.

Table 1: Simulation Parameters

ALIVE NODES:- This is the graph of alive nodes in EPMS and TABU/PSO EPMS protocol. It has been found that the number of nodes alive much more in TP- EPMS protocol. Here, we can see from the graph that the nodes are alive at the round of 2600 in case of EPMS and 3000 in case of TABU/PSO EPMS. 


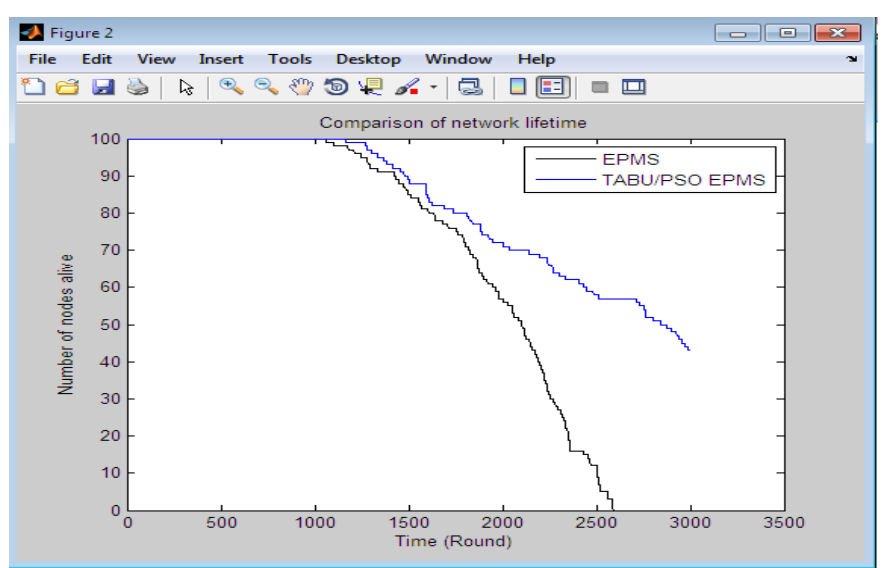

Fig 1.Alive nodes versus rounds

Dead Nodes:- This is the graph of dead nodes in EPMS and TABU/PSO EPMS protocol. The network lifetime can be evaluated by using the number of dead nodes. It has been found that the number of nodes die earlier in EPMS protocol. Here, we can see from the graph that all the nodes are die at the round of 2600 in case of EPMS and 3000 in case of TABU/PSO EPMS .

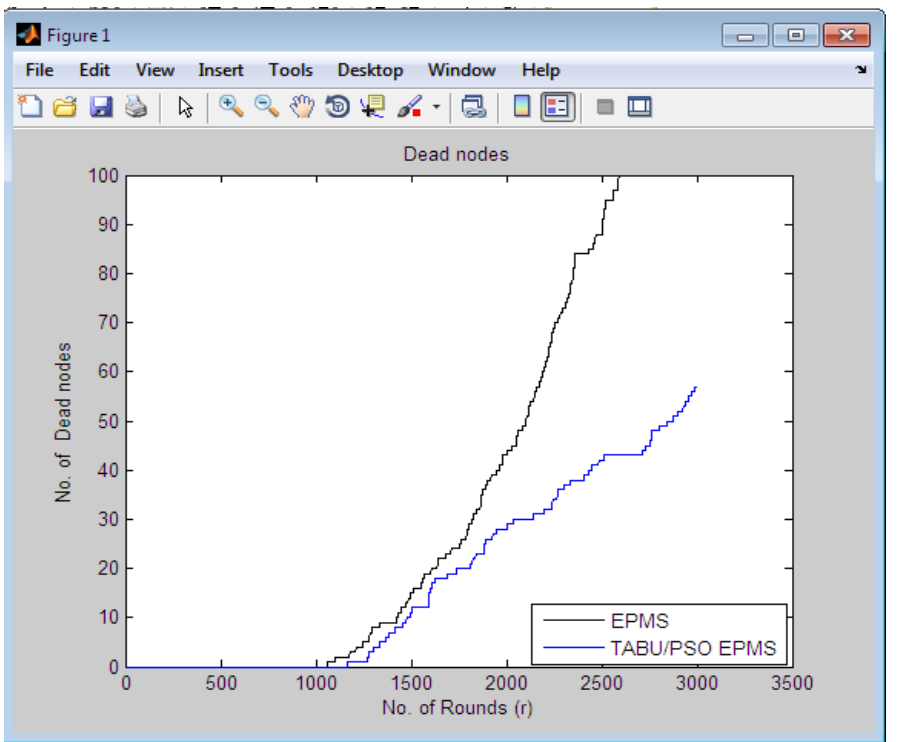

Fig 2. Dead nodes versus rounds

\section{Packets Send to base station:-}

This is the graph of Packet send to base station after simulation. This graph shows the total number of packets send to the base station by the sensor nodes. At the round of 1500, the total number of packets send to base station is 15000 in the case of EPMS protocol and in case of TABU/PSO EPMS , the packets send to base station is 16000 .

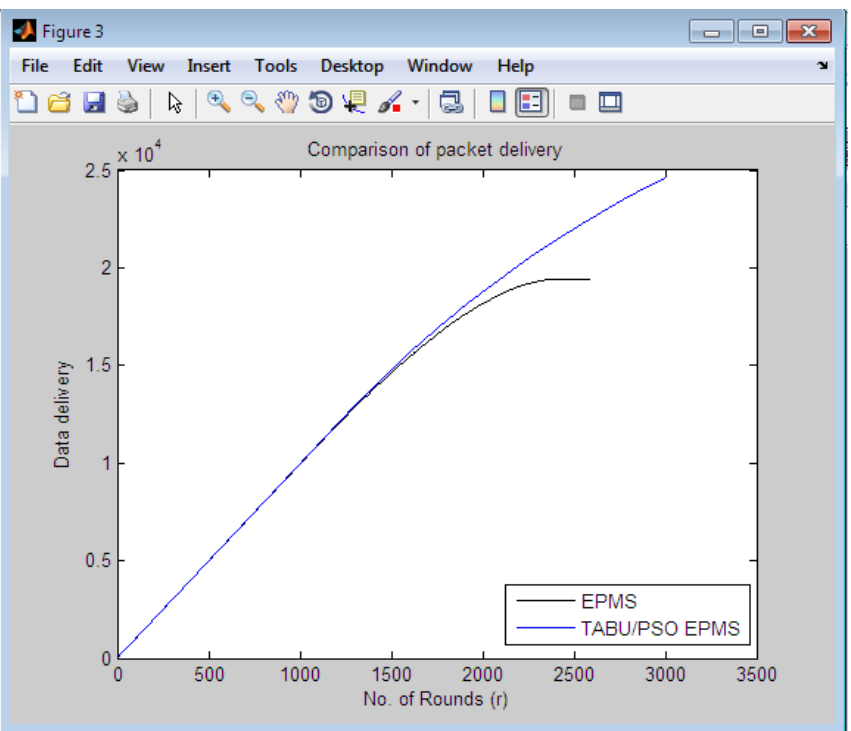

Fig 3.Packet send to base station versus rounds

\section{Remaining Energy:-}

This is the graph of remaining energy, how much energy is left with the rounds. From the graph, we can see the remaining energy with EPMS goes to 2500 rounds, whereas in the case of TABU/PSO EPMS the remaining energy goes to 3000 rounds means more work can be done with TABU/PSO EPMS protocol.

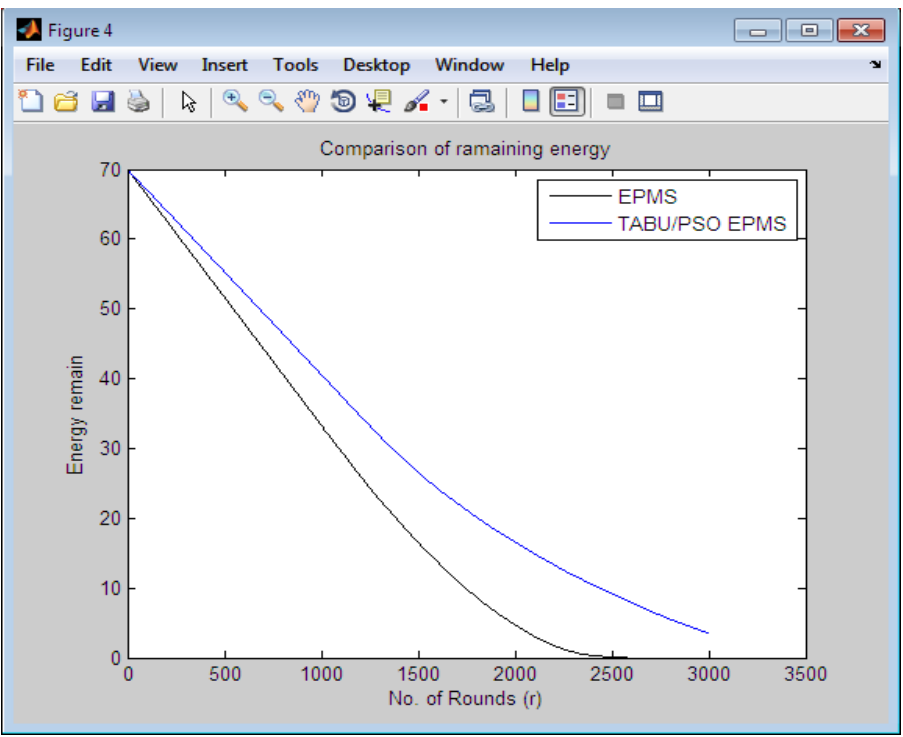

Fig 4. Remaining energy versus rounds

\section{CONCLUSION}

To minimize the energy consumption, we proposed a hybrid routing technique which is called hybrid TABU/PSO EPMS based on swarm intelligence. Hybrid TP- EPMS helps to find out the shortest distance for routing process and thereby enhancement can be done in network lifetime. At the round of 1500 , the total number of packets send to base station is 15000 in the case of EPMS protocol and in case of TABU/PSO EPMS, the packets send to base station is 16000. Similarly, more 
number of nodes are alive in case of TABU/PSO EPMS rather than EPMS For the proposed work, we are using wireless communication and data analysis toolbox of Matlab2013a. The proposed protocol shows the better improvement over existing protocol.

\section{REFERENCES}

[1] W. Heinzelman, A. Chandrakasan, H. Balakrishnan, "Energy-efficient communication protocol for wireless microsensor networks," in: Proceedings of the $33^{\text {rd }}$ Hawaii International Conference on System Sciences, pp.1-10, 2000.

[2] O. Younis, S. Fahmy, "HEED: a hybrid, energyefficient, distributed clustering approach for ad-hoc sensor networks," IEEE Trans. Mob. Comput. 3 (2004)366-379.

[3] S. Lee, J. Yoo, T. Chung, "Distance-based energy efficient clustering in wireless sensor networks," in: Proceedings of the 29th IEEE International Conference on Local Computer Networks, pp.567568, 2004.

[4] Wang. J, Cao. Y et al., “ Particle Swarm optimization based clustering algorithm with mobile sink for WSNs", Future Generation computer system(2016).

[5] Shalli Rani Jyoteesh Malhotra,Rajneesh Talwar,Energy "Efficient chain cooperative routing protocol for WSN", Applied Soft Computing, Elsevier,pp.386-397,2015.

[6] Neeraj, Varsha Sahni, "Review on State-Of-The-Art of PEGASIS Protocol in WSNS", Volume 5 Issue 7, International Journal on Recent and Innovation Trends in Computing and Communication (IJRITCC), ISSN: 2321-8169, PP: 803 - 807,2017.

[7] Liu, W., Lu, K., Wang, J., Xing, G. and Huang, L.(2012), "Performance analysis of wireless sensor networks with mobile sinks", IEEE transactions on vehicular technology, vol. 61, pp.2777-2788.

[8] Glover, F. (1989). Tabu search-part I. ORSA Journal on computing, 1(3), 190-206.

[9] Bala, M., \& Kumar, M. (2019). Randomization of Node Scheme with Optimization in Wireless Sensor Network. International Journal of Advanced Networking and Applications, 10(5), 3999-4006.

[10] Basavaraj, G. N., \& Jaidhar, C. D. (2019). Intersecting Sensor Range Cluster-based Routing Algorithm for Enhancing Energy in WSN. International Journal of Advanced Networking and Applications, 10(4), 39383943. 